\title{
New Mixed Exponential Sums and Their Application
}

\author{
Yu Zhan ${ }^{1}$ and Xiaoxue $\mathrm{Li}^{2}$ \\ ${ }^{1}$ Department of Science, Hetao College, Bayannur 015000, China \\ ${ }^{2}$ School of Mathematics, Northwest University, Xian, Shaanxi 710127, China \\ Correspondence should be addressed to Xiaoxue Li; lxx20072012@163.com
}

Received 6 May 2014; Accepted 23 May 2014; Published 19 June 2014

Academic Editor: Ashraf Zenkour

Copyright (C) 2014 Y. Zhan and X. Li. This is an open access article distributed under the Creative Commons Attribution License, which permits unrestricted use, distribution, and reproduction in any medium, provided the original work is properly cited.

The main purpose of this paper is to introduce a new mixed exponential sums and then use the analytic methods and the properties of Gauss sums to study the computational problems of the mean value involving these sums and give an interesting computational formula and a sharp upper bound estimate for these mixed exponential sums. As an application, we give a new asymptotic formula for the fourth power mean of Dirichlet $L$-functions with the weight of these mixed exponential sums.

\section{Introduction}

Let $q \geq 3$ be an integer, and let $\chi$ be a Dirichlet character $\bmod q$. Then, for any integer $n$, the famous Gauss sums $G(\chi, n)$ are defined as follows:

$$
G(\chi, n)=\sum_{a=1}^{q} \chi(a) \cdot e\left(\frac{n a}{q}\right)
$$

where $e(y)=e^{2 \pi i y}$.

This sum and the other exponential sums (such as Kloosterman sums) play very important role in the study of analytic number theory, and many famous number theoretic problems are closely related to it. For example, the distribution of primes, Goldbach problem, the estimate of character sums, and the properties of Dirichlet $L$-functions are some good examples.

From the properties of characters $\bmod q$, we known that if $\chi$ is a primitive character $\bmod q$, then $G(\chi, n)=\bar{\chi}(n)$. $G(\chi, 1) \equiv \bar{\chi}(n) \cdot \tau(\chi)$, and $|\tau(\chi)|=\sqrt{q}$. Many other properties of $G(\chi, n)$ and $\tau(\chi)$ can also be found in [1-4].

In this paper, we introduce new mixed exponential sums as follows:

$$
\begin{aligned}
G(\chi, c, m, n ; q) \\
\quad=\sum_{a=0}^{q-1} \sum_{b=0}^{q-1} \chi\left(a^{2}+a b+b^{2}+c\right) \cdot e\left(\frac{m a+n b}{q}\right),
\end{aligned}
$$

where $c, m$, and $n$ are any integers.

We will study the arithmetical properties of $G(\chi, c, m, n ; q)$. About this problem, it seems that none has studied it yet; at least we have not seen any related results before. The problem is interesting, because this sum has a close relationship with the general Kloosterman sums, and it is also analogous to famous Gauss sums, so it must have many properties similar to these sums. It can also help us to further understand and study Kloosterman sums and Gauss sums.

The main purpose of this paper is using the analytic method and the properties of Gauss sums to study the fourth power mean of $G(\chi, c, m, n ; p)$ and its upper bound estimate and prove the following three conclusions.

Theorem 1. Let $p$ be an odd prime; let $\chi$ be any nonprincipal character mod $p$. Then, for any integers $c, m$, and $n$, one has the estimate

$$
\left|\sum_{a=0}^{p-1} \sum_{b=0}^{p-1} \chi\left(a^{2}+a b+b^{2}+c\right) \cdot e\left(\frac{m a+n b}{p}\right)\right| \leq 2 p .
$$

Theorem 2. Let $p$ be an odd prime; let $\chi$ be any nonprincipal character $\bmod p$. Then, for any integers $m$ and $n$, one has the identity

$$
\begin{aligned}
\sum_{c=0}^{p-1} & \left|\sum_{a=0}^{p-1} \sum_{b=0}^{p-1} \chi\left(a^{2}+a b+b^{2}+c\right) \cdot e\left(\frac{m a+n b}{p}\right)\right|^{4} \\
& = \begin{cases}3 p^{5}-8 p^{4}, & \text { if } \chi \text { is the Legendre symbol mod } p ; \\
p^{4}(2 p-7), & \text { if } \chi \text { is a complex character } \bmod p .\end{cases}
\end{aligned}
$$


Theorem 3. Let $p$ be an odd prime. Then, for any integers $c$, $m$, and $n$ with $\left(c, m^{2}+n^{2}-m n, p\right) \neq p$, one has the asymptotic formula

$$
\begin{gathered}
\sum_{\substack{\chi \bmod _{\chi} \\
\chi \neq \chi_{0}}}\left|\sum_{a=0}^{p-1} \sum_{b=0}^{p-1} \chi\left(a^{2}+a b+b^{2}+c\right) \cdot e\left(\frac{m a+n b}{p}\right)\right|^{2} \\
\cdot|L(1, \chi)|^{4} \\
=\frac{5}{72} \cdot \pi^{4} \cdot p^{3}+O\left(p^{5 / 2} \cdot \exp \left(\frac{4 \ln \ln p}{\ln p}\right)\right),
\end{gathered}
$$

where $\chi_{0}$ is the principal character $\bmod p,(l, m, n)$ denotes the greatest common divisor of $l, m$, and $n$, and $\exp (y)=e^{y}$.

In Theorem 1, we only discussed the case, in which there exist two variables. For general case (with $k(\geq 3)$ variable), whether there exists a sharp estimate for the sums is an interesting problem.

Let $k \geq 3$; whether there exists an exact computational formula for the $2 k$ th power mean,

$$
\sum_{c=0}^{p-1}\left|\sum_{a=0}^{p-1} \sum_{b=0}^{p-1} \chi\left(a^{2}+a b+b^{2}+c\right) \cdot e\left(\frac{m a+n b}{p}\right)\right|^{2 k},
$$

is also an open problem.

\section{Several Lemmas}

In this section, we will give several lemmas, which are necessary in the proof of our theorems. Hereinafter, we will use many properties of character sums, Kloosterman sums, and Gauss sums; all of these can be found in [1,5-7], so they will not be repeated here. First, we have the following.

Lemma 4. Let $p$ be an odd prime; then, for any integers $c, m$, and $n$, one has the identity

$$
\begin{aligned}
& \left|\sum_{a=0}^{p-1} \sum_{b=0}^{p-1} \chi\left(a^{2}+a b+b^{2}+c\right) \cdot e\left(\frac{m a+n b}{p}\right)\right| \\
& \quad=\sqrt{p} \cdot\left|\sum_{r=1}^{p-1} \bar{\chi}(r) \cdot e\left(\frac{c r-\overline{4} \bar{r}\left(m^{2}+n^{2}-m n\right)}{p}\right)\right|,
\end{aligned}
$$

where $\bar{r}$ denotes the solution of the congruence equation $r \cdot x \equiv$ $1 \bmod p$.

Proof. If $(n, p)=1$, then, from the properties of Gauss sums and quadratic residue $\bmod p$, we have

$$
\begin{aligned}
\sum_{a=0}^{p-1} e\left(\frac{n a^{2}}{p}\right) & =1+\sum_{a=1}^{p-1} e\left(\frac{n a^{2}}{p}\right) \\
& =1+\sum_{a=1}^{p-1}\left(1+\left(\frac{a}{p}\right)\right) \cdot e\left(\frac{n a}{p}\right)
\end{aligned}
$$

$$
\begin{aligned}
& =\sum_{a=0}^{p-1} e\left(\frac{n a}{p}\right)+\sum_{a=1}^{p-1}\left(\frac{a}{p}\right) \cdot e\left(\frac{n a}{p}\right) \\
& =\left(\frac{n}{p}\right) \sum_{a=1}^{p-1}\left(\frac{a}{p}\right) \cdot e\left(\frac{a}{p}\right)=\left(\frac{n}{p}\right) \cdot \tau\left(\chi_{2}\right),
\end{aligned}
$$

where $\chi_{2}=(* / p)$ denotes the Legendre symbol.

Since $\chi$ is a nonprincipal Dirichlet character $\bmod p$, from (8), the properties of Gauss sums, and complete residue system $\bmod p$, we have

$$
\begin{aligned}
& \sum_{a=0}^{p-1} \sum_{b=0}^{p-1} \chi\left(a^{2}+a b+b^{2}+c\right) \cdot e\left(\frac{m a+n b}{p}\right) \\
& =\frac{1}{\tau(\bar{\chi})} \cdot \sum_{a=0}^{p-1} \sum_{b=0}^{p-1} \sum_{r=1}^{p-1} \bar{\chi}(r) e\left(\frac{r\left(a^{2}+a b+b^{2}+c\right)}{p}\right) \\
& \cdot e\left(\frac{m a+n b}{p}\right) \\
& =\frac{1}{\tau(\bar{\chi})} \\
& \cdot \sum_{r=1}^{p-1} \bar{\chi}(r) \sum_{a=0}^{p-1} \sum_{b=0}^{p-1} e\left(\frac{r a^{2}+(b r+m) a+r b^{2}+n b+c r}{p}\right) \\
& =\frac{1}{\tau(\bar{\chi})} \sum_{r=1}^{p-1} \bar{\chi}(r) \\
& \times \sum_{b=0}^{p-1} \sum_{a=0}^{p-1} e\left(\frac{\overline{4} r(2 a+b+\bar{r} m)^{2}+r b^{2}+r c+n b-\overline{4} r(b+\bar{r} m)^{2}}{p}\right) \\
& =\frac{1}{\tau(\bar{\chi})} \cdot \sum_{r=1}^{p-1} \bar{\chi}(r) \\
& \times \sum_{b=0}^{p-1} \sum_{a=0}^{p-1} e\left(\frac{r a^{2}+3 r(\overline{2} b)^{2}+(2 n-m) \overline{2} b+r c-\overline{4} \bar{r} m^{2}}{p}\right) \\
& =\frac{\tau\left(\chi_{2}\right)}{\tau(\bar{\chi})} \cdot \sum_{r=1}^{p-1} \bar{\chi}(r) \chi_{2}(r) \\
& \times \sum_{b=0}^{p-1} e\left(\frac{3 r \overline{4}(b+\overline{3} \bar{r}(2 n-m))^{2}+r c-\overline{4} \bar{r} m^{2}-\overline{12} \bar{r}(2 n-m)^{2}}{p}\right) \\
& =\frac{\tau\left(\chi_{2}\right)}{\tau(\bar{\chi})} \cdot \sum_{r=1}^{p-1} \bar{\chi}(r) \chi_{2}(r) \\
& \times \sum_{b=0}^{p-1} e\left(\frac{3 r b^{2}+r c-\overline{4} \bar{r}\left(m^{2}+n^{2}-m n\right)}{p}\right) \\
& =\frac{\tau^{2}\left(\chi_{2}\right)}{\tau(\bar{\chi})} \cdot \chi_{2}(3) \cdot \sum_{r=1}^{p-1} \bar{\chi}(r) e\left(\frac{c r-\overline{4} \bar{r}\left(m^{2}+n^{2}-m n\right)}{p}\right) \text {. }
\end{aligned}
$$


For any nonprincipal character $\chi \bmod p$, we have $|\tau(\chi)|=$ $\sqrt{p}$. So, from (9) and noting that $\left|\bar{\chi}_{2}(3)\right|=1$, we have

$$
\begin{aligned}
& \left|\sum_{a=0}^{p-1} \sum_{b=0}^{p-1} \chi\left(a^{2}+a b+b^{2}+c\right) \cdot e\left(\frac{m a+n b}{p}\right)\right| \\
& \quad=\sqrt{p} \cdot\left|\sum_{r=1}^{p-1} \bar{\chi}(r) \cdot e\left(\frac{c r-\overline{4} \bar{r}\left(m^{2}+n^{2}-m n\right)}{p}\right)\right| .
\end{aligned}
$$

This proves Lemma 4.

Lemma 5. Let $p$ be an odd prime; let $\chi$ be any nonprincipal character $\bmod p$. Then, for any integers $m$ and $n$, one has the estimate

$$
\sum_{a=1}^{p-1} \chi(a) \cdot e\left(\frac{m a+n \bar{a}}{p}\right) \leq 2 \sqrt{p}
$$

Proof. Since $\chi$ is a nonprincipal character $\bmod p$, if $p \mid m$ and $p \mid n$, then

$$
\sum_{a=1}^{p-1} \chi(a) \cdot e\left(\frac{m a+n \bar{a}}{p}\right)=\sum_{a=1}^{p-1} \chi(a)=0 .
$$

If $p \mid m$ and $(p, n)=1$ or $p \mid n$ and $(p, m)=1$ or $(m n, p)=1$, then, from the results of Weil [8], Malyšev [7], and Estermann [6], with some minor modifications, we can deduce the estimate

$$
\left|\sum_{a=1}^{p-1} \chi(a) \cdot e\left(\frac{m a+n \bar{a}}{p}\right)\right| \leq 2 \sqrt{p} \cdot(m, n, p)^{1 / 2}=2 \sqrt{p},
$$

where $(m, n, p)$ denotes the greatest common divisor of $m, n$, and $p$.

Now Lemma 5 follows from (12) and (13).

Lemma 6. Let $p$ be an odd prime; then, for any integer $n$ with $(n, p)=1$, one has the calculating formula

$$
\begin{aligned}
& \sum_{m=1}^{p-1}\left|\sum_{a=1}^{p-1} \chi(a) \cdot e\left(\frac{m a+n \bar{a}}{p}\right)\right|^{4} \\
& =\left\{\begin{array}{c}
2 p^{3}-3 p^{2}-3 p-1, \\
\text { if } \chi \text { is the principal character mod } p ; \\
3 p^{3}-8 p^{2} \\
\text { if } \chi \text { is the Legendre's symbol } \bmod p ; \\
p^{2}(2 p-7), \\
\text { if } \chi \text { is a non-real character } \bmod p .
\end{array}\right.
\end{aligned}
$$

Proof. See [9] or Corollary 2 of [10].

Lemma 7. Let $p$ be an odd prime and let $\chi$ be the Dirichlet character $\bmod p$. Then one has the estimate

$$
\left.\sum_{a=1}^{p-1}\left|\sum_{\substack{\chi \bmod p \\ \chi \neq \chi_{0}}} \chi(a)\right| L(1, \chi)\right|^{4} \mid=O\left(p \cdot \exp \left(\frac{4 \ln \ln p}{\ln p}\right)\right) .
$$

Proof. See Lemma 5 of [11].

\section{Proof of the Theorems}

In this section, we will complete the proof of our theorems. First we prove Theorem 1. In fact, from Lemmas 4 and 5, we may immediately deduce the estimate

$$
\begin{aligned}
& \left|\sum_{a=0}^{p-1} \sum_{b=0}^{p-1} \chi\left(a^{2}+a b+b^{2}+c\right) \cdot e\left(\frac{m a+n b}{p}\right)\right| \\
& \quad=\sqrt{p} \cdot\left|\sum_{r=1}^{p-1} \bar{\chi}(r) \cdot e\left(\frac{c r-\overline{4} \bar{r}\left(m^{2}+n^{2}-m n\right)}{p}\right)\right| \leq 2 p .
\end{aligned}
$$

This proves Theorem 1 .

Theorem 2 follows from Lemmas 4 and 6. In fact, from these two lemmas, we have

$$
\begin{aligned}
& \sum_{c=1}^{p-1}\left|\sum_{a=0}^{p-1} \sum_{b=0}^{p-1} \chi\left(a^{2}+a b+b^{2}+c\right) \cdot e\left(\frac{m a+n b}{p}\right)\right|^{4} \\
& =p^{2} \cdot \sum_{c=1}^{p-1}\left|\sum_{r=1}^{p-1} \bar{\chi}(r) \cdot e\left(\frac{c r-\overline{4} \bar{r}\left(m^{2}+n^{2}-m n\right)}{p}\right)\right|^{4} \\
& = \begin{cases}3 p^{5}-8 p^{4}, & \text { if } \chi \text { is the Legendre symbol } \bmod p \\
p^{4}(2 p-7), & \text { if } \chi \text { is a complex character } \bmod p .\end{cases}
\end{aligned}
$$

This proves Theorem 2 .

Now, we prove Theorem 3. Note that the asymptotic formula is

$$
\sum_{\substack{\chi \bmod p \\ \chi \neq \chi_{0}}}|L(1, \chi)|^{4}=\frac{5}{72} \cdot \pi^{4} \cdot p+O\left(\exp \left(\frac{4 \ln \ln p}{\ln p}\right)\right),
$$

and the estimate for Kloosterman sums (see [6]) is as follows:

$$
\sum_{a=1}^{p-1} e\left(\frac{m a+n \bar{a}}{p}\right) \ll \sqrt{p} \cdot(m, n, p)^{1 / 2}
$$

from Lemmas 4 and 7 and the method of proving the theorem in [11], we have

$$
\begin{aligned}
& \sum_{\substack{\chi \bmod _{\chi} \\
\chi \neq \chi_{0}}}\left|\sum_{a=0}^{p-1} \sum_{b=0}^{p-1} \chi\left(a^{2}+a b+b^{2}+c\right) \cdot e\left(\frac{m a+n b}{p}\right)\right|^{2} \\
& \cdot|L(1, \chi)|^{4} \\
& =p \cdot \sum_{\substack{\chi \bmod _{p} \\
\chi \neq \chi_{0}}}\left|\sum_{r=1}^{p-1} \bar{\chi}(r) \cdot e\left(\frac{c r-\overline{4} \bar{r}\left(m^{2}+n^{2}-m n\right)}{p}\right)\right|^{2}
\end{aligned}
$$

$$
\cdot|L(1, \chi)|^{4}
$$




$$
\begin{aligned}
& p \cdot \sum_{r=1}^{p-1} \sum_{s=1}^{p-1} e\left(\frac{c(r-s)-\overline{4}(\bar{r}-\bar{s})\left(m^{2}+n^{2}-m n\right)}{p}\right) \\
& \sum_{\substack{\chi \bmod _{p} \\
\chi \neq \chi_{0}}} \bar{\chi}(r \bar{s})|L(1, \chi)|^{4} \\
& p \cdot \sum_{r=1}^{p-1} \sum_{s=1}^{p-1} e\left(\frac{c s(r-1)-\overline{4} \bar{s}(\bar{r}-1)\left(m^{2}+n^{2}-m n\right)}{p}\right) \\
& \sum_{\substack{\chi \bmod p \\
\chi \neq \chi_{0}}} \bar{\chi}(r)|L(1, \chi)|^{4} \\
& =p(p-1) \sum_{\substack{\chi \bmod p \\
\chi \neq \chi_{0}}}|L(1, \chi)|^{4} \\
& +O\left(\left.p^{3 / 2} \cdot \sum_{r=2}^{p-1}\left|\sum_{\substack{\chi \bmod _{\chi} p \\
\chi \neq \chi_{0}}} \bar{\chi}(r)\right| L(1, \chi)\right|^{4} \mid\right) \\
& =\frac{5}{72} \cdot \pi^{4} \cdot p^{3}+O\left(p^{5 / 2} \cdot \exp \left(\frac{4 \ln \ln p}{\ln p}\right)\right) \text {. }
\end{aligned}
$$

This completes the proof of Theorem 3.

\section{Conflict of Interests}

The authors declare that they have no conflict of interests.

\section{Authors' Contribution}

Zhan $\mathrm{Yu}$ obtained the theorems and completed the proof. Li Xiaoxue corrected and improved the final version. Both authors read and approved the final paper.

\section{Acknowledgments}

The authors would like to thank the referee for his very helpful and detailed comments, which have significantly improved the presentation of this paper. This work is supported by the P. S. F. (2013JZ001) and N. S. F. (11371291) of China.

\section{References}

[1] T. M. Apostol, Introduction to Analytic Number Theory, Springer, New York, NY, USA, 1976.

[2] C. D. Pan and C. B. Pan, Goldbach Conjecture, Science Press, Beijing, China, 1992.

[3] H. Davenport, Multiplicative Number Theory, vol. 1966, Markham, 1967.

[4] K. F. Ireland and M. I. Rosen, A Classical Introduction to Modern Number Theory, vol. 84, Springer, New York, NY, USA, 1982.

[5] S. Chowla, On Kloosterman's Sum, vol. 40 of Volume 1967 of Forhandlinger, F. Bruns, 1968.
[6] T. Estermann, “On Kloostermann's sums," Mathematica, vol. 8, pp. 83-86, 1961.

[7] A. V. Malyšev, "A generalization of Kloosterman sums and their estimates," Vestnik Leningrad University, vol. 15, no. 13, pp. 5975, 1960 (Russian).

[8] A. Weil, "On some exponential sums," Proceedings of the National Academy of Sciences of the United States of America, vol. 34, pp. 204-207, 1948.

[9] W. Zhang, "On the fourth power mean of the general Kloosterman sums," Indian Journal of Pure and Applied Mathematics, vol. 35, no. 2, pp. 237-242, 2004.

[10] J. H. Li and Y. N. Liu, "Some new identities involving Gauss sums and general Kloosterman sums," Acta Mathematica Sinica, vol. 56, no. 3, pp. 413-418, 2013 (Chinese).

[11] W. Zhang, Y. Yi, and X. He, "On the $2 k$-th power mean of Dirichlet $L$-functions with the weight of general Kloosterman sums," Journal of Number Theory, vol. 84, no. 2, pp. 199-213, 2000 . 


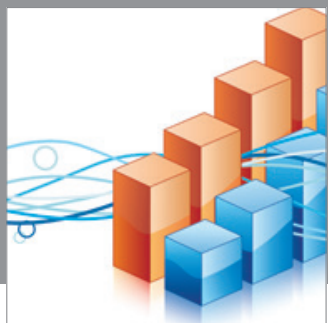

Advances in

Operations Research

mansans

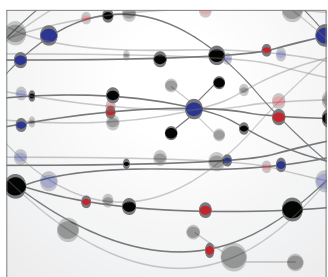

The Scientific World Journal
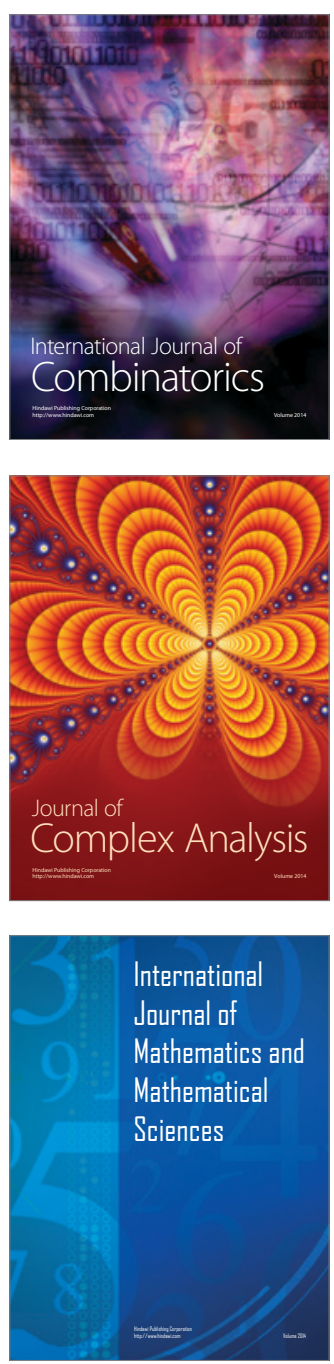
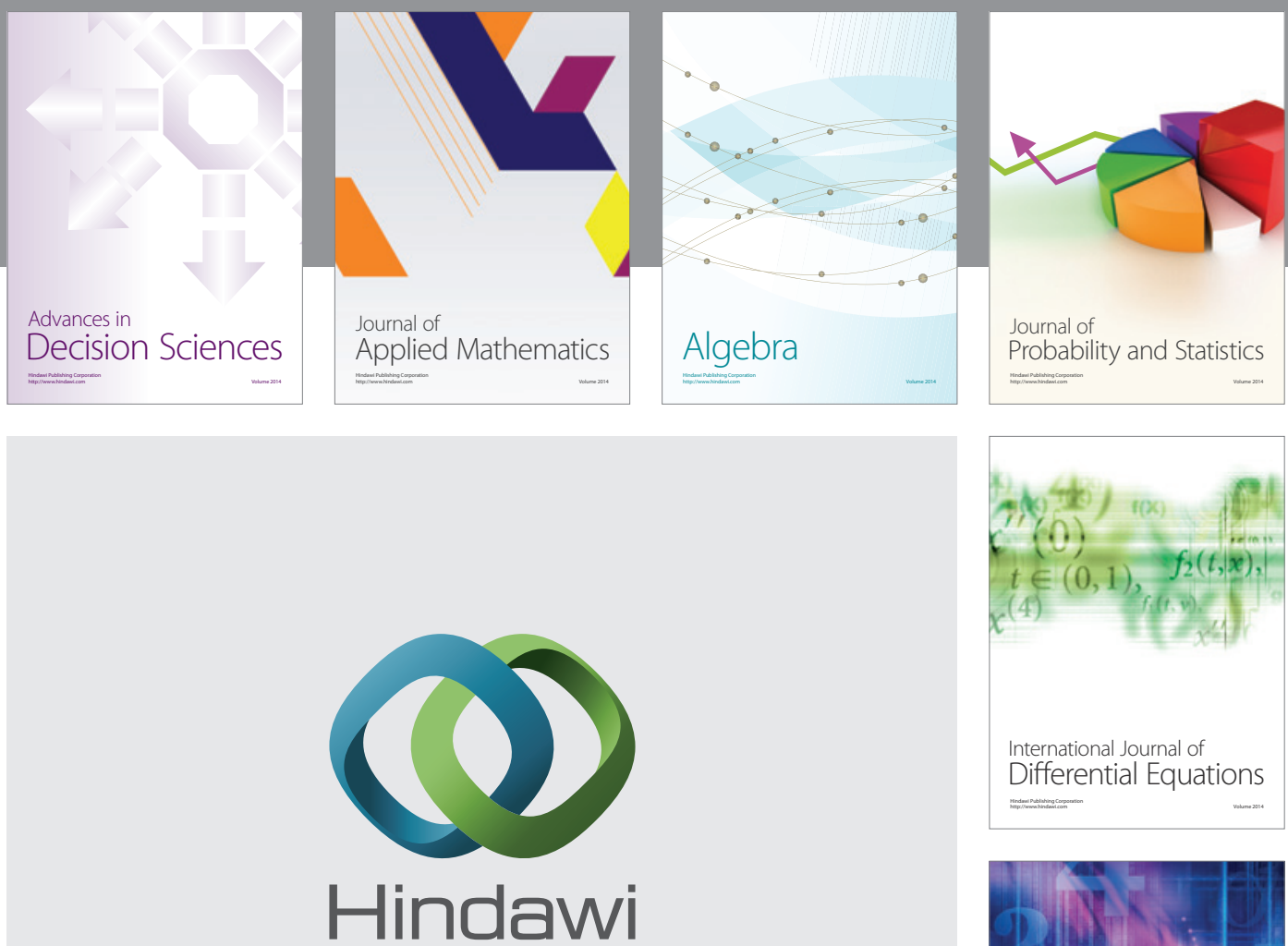

Submit your manuscripts at http://www.hindawi.com
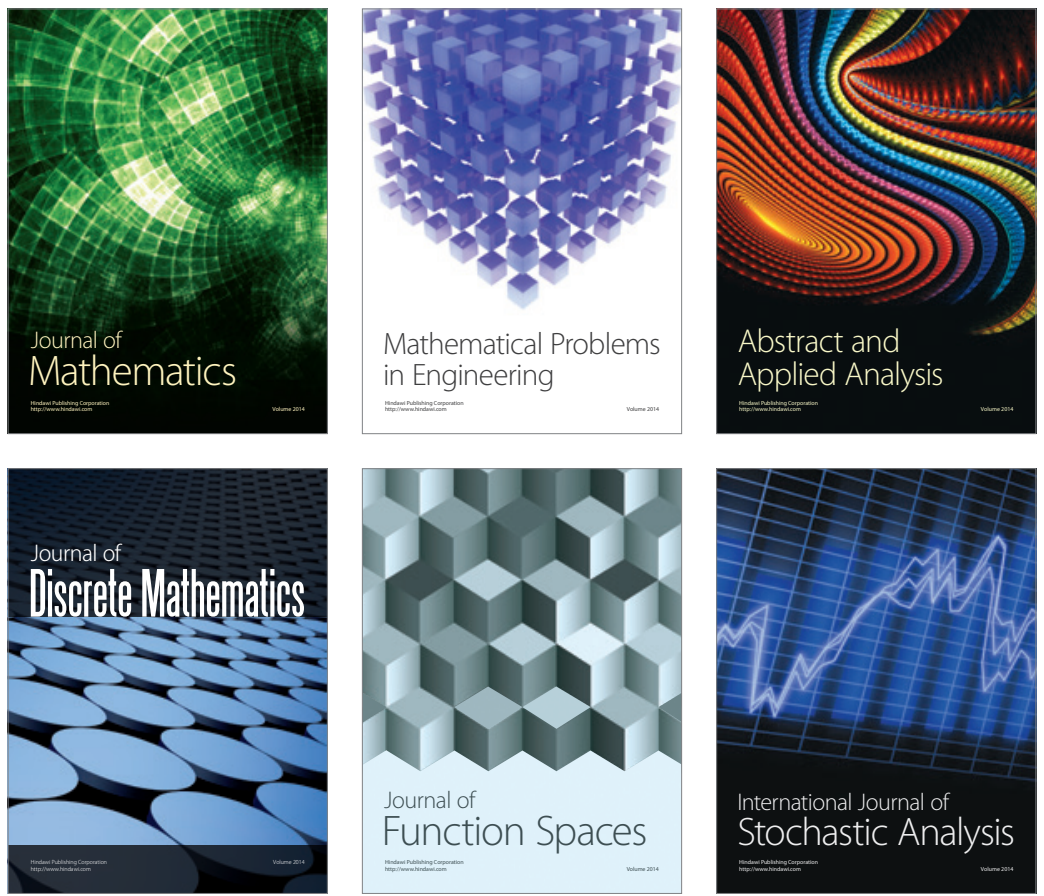

Journal of

Function Spaces

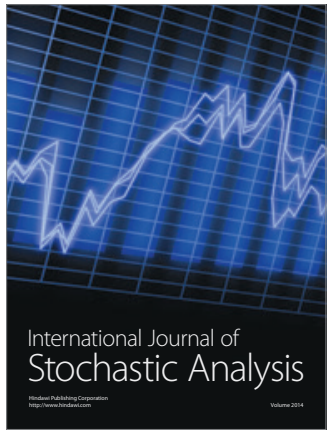

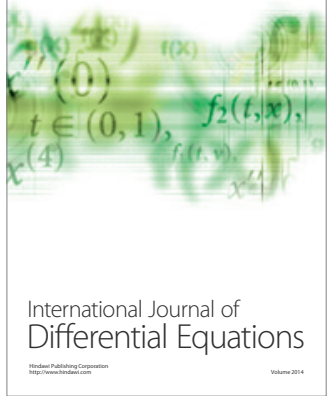
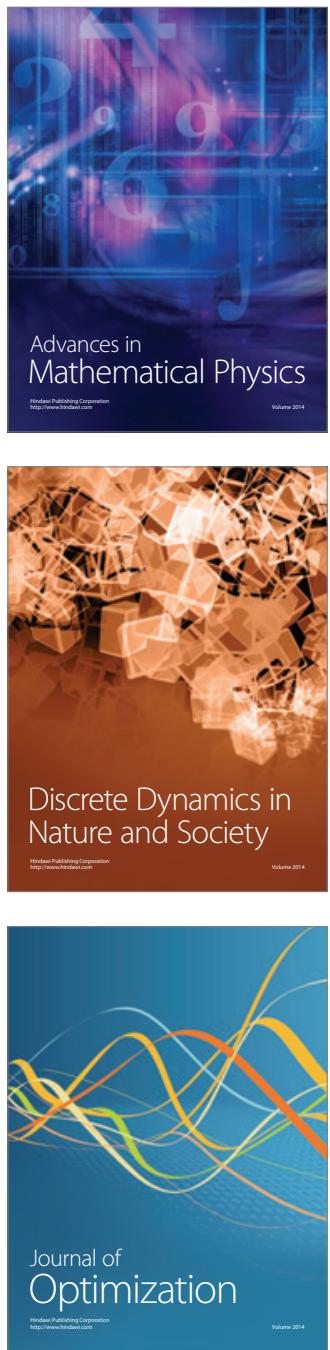